\title{
On extreme geomagnetic storms
}

\author{
Consuelo Cid*, Judith Palacios, Elena Saiz, Antonio Guerrero, and Yolanda Cerrato \\ Space Research Group - Space Weather, Departamento de Física y Matemáticas, Universidad de Alcalá, Alcalá de Henares, Spain \\ *Corresponding author: consuelo.cid@uah.es
}

Received 17 March 2014 / Accepted 13 September 2014

\begin{abstract}
Extreme geomagnetic storms are considered as one of the major natural hazards for technology-dependent society. Geomagnetic field disturbances can disrupt the operation of critical infrastructures relying on space-based assets, and can also result in terrestrial effects, such as the Quebec electrical disruption in 1989. Forecasting potential hazards is a matter of high priority, but considering large flares as the only criterion for early-warning systems has demonstrated to release a large amount of false alarms and misses. Moreover, the quantification of the severity of the geomagnetic disturbance at the terrestrial surface using indices as Dst cannot be considered as the best approach to give account of the damage in utilities. High temporal resolution local indices come out as a possible solution to this issue, as disturbances recorded at the terrestrial surface differ largely both in latitude and longitude. The recovery phase of extreme storms presents also some peculiar features which make it different from other less intense storms. This paper goes through all these issues related to extreme storms by analysing a few events, highlighting the March 1989 storm, related to the Quebec blackout, and the October 2003 event, when several transformers burnt out in South Africa.
\end{abstract}

Key words. geomagnetic storm - space weather - geomagnetic indices

\section{Introduction}

Nowadays, geomagnetic storms are recognised as natural hazards. Scientific community and governmental entities are aware about the possibility of severe disturbances of terrestrial environment caused by solar activity may threaten critical infrastructure. However, the underlying physical mechanisms remain poorly understood. As a result, the scientific community is far from being able to accurately forecast severe storms without releasing a large amount of false alarms, which leads to reduce trust in the procedure by the users community.

Space weather predictions are usually obtained by database-calibrated models. The very low statistical significance of severe storms is a key issue for model calibration, that is fed by data mainly from moderate to intense disturbances, due to the lack of severe storms in the sample (Srivastava 2005; Kataoka 2013; Yermolaev et al. 2013).

Carrington (1859) connected "two patches of intensely bright and white light" in a large solar spot with "a moderate but very marked magnetic disturbance of short duration" in Kew magnetic records followed by the commencement of a great magnetic storm. This time was the first when large flares were associated with extreme terrestrial disturbances. However, magnetic records at terrestrial surface may not show remarkable disturbances after large flares, as happened on 5 December 2006.

Since Gosling (1993) set out of focus the role of solar flares in the paradigm of cause-effect of non-recurrent geomagnetic storms, giving the central role to coronal mass ejections (CMEs), this kind of solar activity has increased its relevance in space weather studies. In the identification of the solar precursor of geomagnetic activity, interplanetary counterparts of solar ejections have played a major role, as linking every chain along the Sun-to-Earth line is not an easy task (Rodriguez et al. 2009).
Gonzalez et al. (2007) and Echer et al. (2008) found that the dominant interplanetary structures which triggered the most intense geomagnetic storms are magnetic clouds or sheath fields. Several studies (e.g., Wang et al. 2003; Xie et al. 2006; Zhang et al. 2007; Yermolaev \& Yermolaev 2008, and references therein) showed multiple interacting magnetic clouds, as a result of the release of successive CMEs, were involved in a significant number of intense storms. From the analysis of the whole scenario of the solar-interplanetary event leading to the eleven superintense (Dst $\leq-250 \mathrm{nT})$ geomagnetic storms that occurred during solar cycle 23, Cid et al. (2008) concluded that interaction among multiple magnetic clouds or even fast streams from solar coronal holes (hereafter $\mathrm{CHs}$ ) were involved. In some cases, magnetic clouds alone were observed, but in these cases, interplanetary data showed clearly the overtaking of successive structures in their travel far from the solar surface.

A key issue for interplanetary disturbances to lead the occurrence of extreme events is the direction of the interplanetary magnetic field vector and the solar wind speed. Gonzalez et al. (2011) showed that for superstorms, velocity and southward magnetic field reached very large values of about $800 \mathrm{~km} \mathrm{~s}^{-1}$ and $42 \mathrm{nT}$, respectively.

In this scenario, a CME observed by STEREO-A in 23 July 2012 with an initial speed of $2500 \mathrm{~km} \mathrm{~s}^{-1}$, but directed away from the Earth, renewed interest in the study of extreme geomagnetic storms due to their potential impact (Baker et al. 2013; Russell et al. 2013; Ngwira et al. 2013a; Ngwira et al. 2014). The average transit speed of the leading edge of the magnetic cloud of $1910 \mathrm{~km} \mathrm{~s}^{-1}$ and a peak magnetic field strength of $109 \mathrm{nT}$, one of the largest records near $1 \mathrm{AU}$, evidenced that extreme space weather conditions can happen during modest solar activity cycle (Russell et al. 2013). Two flux ropes appeared at interplanetary STEREO-A, as 
evidenced rotations of the interplanetary magnetic field. Careful analysis of the in-situ signatures indicate that the mechanism of creating that extremely strong magnetic field was $\mathrm{CME}-\mathrm{CME}$ interaction, suggesting as a possible scenario two CMEs with a shock driven by the second CME overtaking the first CME from behind before the direct compression of CME2 occurred (Liu et al. 2014).

The events described above evidence that interaction of flux ropes with other interplanetary transients (shocks, fast streams, or other flux ropes) plays a central role in the paradigm of cause-effect of severe geomagnetic disturbances. But this statement is based on interplanetary measurements and the solar cause of extreme space weather also needs to be addressed to accurately forecast well in advance.

Several studies have addressed the relation between filament eruptions, flares and CMEs (e.g., Jing et al. 2004), reporting that the percentage of CMEs associated to eruptive filaments ranges from $43 \%$ to $54 \%$, while the filament-flare association is extremely variable, from $27 \%$ to $95 \%$, depending on the filament's behaviour. On the other hand, an atypical but significant case can be considered (Démoulin et al. 2002), where a large number of CMEs can be mistaken and misidentified (by association) to the only important active region in the solar hemisphere facing Earth.

In this scenario, a key question to be solved is: What therefore are the solar triggers of extreme storms?

Indices such as Dst are used to assess magnetic storms severity. Thus, a Dst index below $-50 \mathrm{nT}$ is indicative of moderate disturbance, which turns to intense when $-100 \mathrm{nT}$ threshold is passed (Gonzalez et al. 1994) and superintense or extreme if Dst reaches less than $-250 \mathrm{nT}$ (Echer et al. 2008). For the derivation of the Dst index, the horizontal component, $H$, of the geomagnetic field recorded at four magnetic observatories (Hermanus, Kakioka, Honolulu, and San Juan) is used. These records are averaged and weighted through the latitude cosine to get Dst, which is supposed to provide a quantitative measure of geomagnetic disturbance that will be correlated with other solar and geophysical parameters.

The Carrington event of 1859, the March 1989 storm responsible for the Quebec power outage or the October 2003 storm threatening the electrical grid in South Africa and Sweden cannot be missed in the short list of historical records of extreme geomagnetic storms due to their consequences for society. All these three storms had consequences in infrastructures, and all of them were superintense as seen by $D s t$ index. Indeed, Dst peaked below $-250 \mathrm{nT}$ for these events according to the classification mentioned above. Thus, the 29 October 2003 storm Dst reached $-383 \mathrm{nT}$; for the 13-14 March 1989 storm it reached $-589 \mathrm{nT}$, and for the Carrington event it was estimated by Lakhina et al. (2005) as $-1760 \mathrm{nT}$, i.e. approximately three times more intense than the Quebec storm. Other estimations for the minimum $D s t$ value reached during the Carrington storm have been done using the record of the event at Colaba observatory, that is, a decrease in $H$ of $-1600 \mathrm{nT}$ (Tsurutani et al. 2003). The estimated Dst minimum value ranges from about $-2000 \mathrm{nT}$ (Siscoe 1979) to $-685 \mathrm{nT}$ (Cid et al. 2013). This last estimation makes the Carrington storm comparable to the Quebec storm in 1989.

On the other hand, the storm occurred in July 2000, known as the Bastille event, is considered extreme since Dst reached a minimum value of $-301 \mathrm{nT}$, although no failure in infrastructures was reported. This fact led us to propose that, in addition to a physical-based classification scheme for storms, an effectbased scale is needed, similar to earthquake scales. The main interest regarding vulnerability is to define the term extreme storm as the storm which results in severe damages. Indices as Dst are not scaled to damage quantification. Since 1989, the minimum values Dst reached were $-422 \mathrm{nT}$ on 20 November 2003 and $-387 \mathrm{nT}$ on 31 March 2001. Both storms were therefore more intense than the October 2003 storm $(D s t=-383 \mathrm{nT})$, but no damage in utilities was reported. Indeed Dst reached below $-350 \mathrm{nT}$ also in November 1991 and November 2004, and these events should not be labeled as severe due to the lack of damage.

Furthermore, Love \& Gannon (2010) reported for the 29 October 2003 storm a large asymmetry in the low-latitude disturbance with longitude, being the largest disturbance difference value of $867 \mathrm{nT}$ at 06:57 UT. However, this asymmetry is even larger at mid- and high-latitudes and might be the physical reason for the outages threatening the electrical grid in Europe or Africa, being unnoticed for utilities in America. The disturbance dependence on the station latitude was also considered by Tyasto et al. (2009) as a possible interpretation for the differences in the measurements registered at several Russian stations during the event in September 1859. The fact that local disturbances differ significantly from one to another evidences that quantification of local disturbances is a key issue to assess damage in infrastructures.

As far as the local or global character of damages is concerned, there is a lot of information regarding the October 2003 storm. Sweden experienced a blackout affecting around 50,000 customers but minor power grid disturbances were experienced in North America. ${ }^{1}$ On the other hand, twelve transformers in South Africa suffered damage and required removal from service, what evidenced that mid-latitude locations (Gaunt \& Coetzee 2007; Thomson et al. 2010) - and even low-latitudes (Yizengaw et al. 2013) - are not immune from space weather disturbances. But Sweden and South Africa have similar geographic longitude.

In this scenario, the questions are obvious: could new indicators be defined to quantify the disturbance at terrestrial surface according to potential consequences for infrastructures? What about the influence of longitude in the severity of the geomagnetic disturbance?

The recovery phase of extreme storms also deserves some attention. The extremely fast recovery during the Carrington storm was recently modeled by Cid et al. (2013) using a hyperbolic decay function. The early recovery phase cannot be reproduced by a unique exponential law. Moreover, the more intense the storm is, the faster the magnetosphere recovers at its first stages. For the first time, Aguado et al. (2010) proposed the hyperbolic function to explain the complete recovery phase of intense storms, based on the Dst index. Let us recount that the Dst provided in the literature for the Carrington storm was obtained from magnetic records from just one observatory. Does it mean that local magnetic disturbances recover also following a hyperbolic law?

The increasing awareness over severe consequences related to extreme space weather disturbances led us to try to answer in this paper the questions posed above by analysing data available of some extreme geomagnetic storms, as they are keyquestions that need to be properly answered in order to achieve efficient forecasting space weather tools. Section 2 is devoted

\footnotetext{
${ }^{1}$ http://www.lloyds.com/news-and-insight/risk-insight/reports/ space/space-weather
} 
to solar triggers, Section 3 is focused on local magnetic records and geomagnetic indices, and Section 4 examines the recovery phase. Finally, Section 5 draws the conclusions of this work and discusses outstanding problems and challenges to be addressed in future studies.

\section{Solar triggers of extreme geomagnetic storms}

To understand the Sun-to-Earth chain, it is fundamental to look for triggers at the first link of the chain. A historical reference is Carrington (1859), who witnessed the largest registered geomagnetic storm and related it to a white-light flare in the Sun from a very large active region, as seen in the photosphere. With this event sequence (including his hesitation expressed as "one swallow does not make a summer"), he set the foundational ideas of space weather.

Solar active regions usually reach kilogauss values in their magnetic field. The 3D topology of these regions can favour the creation of sheared arcades and flux ropes. A flux rope can emerge by reconnection (van Ballegooijen \& Martens 1989) and different physical mechanisms for instability and eruption have been proposed (e.g., see Aulanier 2014, for a review). Eruptions can be confined, when they are not associated with coronal mass ejections; or eruptive, when they are (Svestka \& Cliver 1992). The eruptive events can display some other signatures, as dimming in EUV images and waves (Zhukov \& Auchère 2004), flaring and jets. The eruption can be defined as "reconnection-induced eruption" or "eruption-induced reconnection" (Kusano et al. 2012), depending whether the flare was first and next the ejection, or the other way round.

When eruptive processes happen, a CME can be released. CMEs and all the subsequent interactions in the interplanetary medium can convert ICMEs into geoeffective cases. In this Section, a review of the solar causes of the events of 2000 and 2003 mentioned above, and a case from 2005 will be analysed. The first two aforementioned events are relevant because their geomagnetic counterparts were extreme storms. In the last mentioned case (2005), the Dst index did not reach $-200 \mathrm{nT}$ but a sharp drop in less than $1 \mathrm{~h}$ was registered, and this fact can have further effects due to geomagnetically induced currents (GICs).

The solar events that triggered the geomagnetic storms of July 2000 and October 2003 displayed a whole amount of features corresponding to a chain of CMEs, that we describe below.

In the case of the geomagnetic storm on 15 July 2000 ("The Bastille Storm"), only one active region, NOAA 09077, produced 3 full-halo CMEs on 11, 12 and 14 July. The plane-of-sky speed of the CMEs ranged from 1000 to $1700 \mathrm{~km} \mathrm{~s}^{-1}$ (Cerrato et al. 2012). The associated X-flares were on 11 July at 12:12 UT (X1), 12 July at 10:18 UT (X1.9) and 14 July at 10:03 UT (X5.7). The active region proper motion, large shear and polarity squeezing are the main magnetic features that led to those flares (Deng et al. 2001).

The "Halloween Storm" (31 October 2003) was produced by a series of full-halo CMEs. Previously to the storm, the active region (AR) NOAA 10486 released a partial-halo CME on 26 October at 06:54 UT, and another partial-halo CME at 17:54 UT from AR 10484. Both CMEs were related to X1.2 flares and they disturbed the interplanetary medium.

The actual CME chain provoking the storm is the following: a full-halo CME was emitted by AR 10486 on 28 October at 11:30 UT (with one of the largest flares ever recorded, a

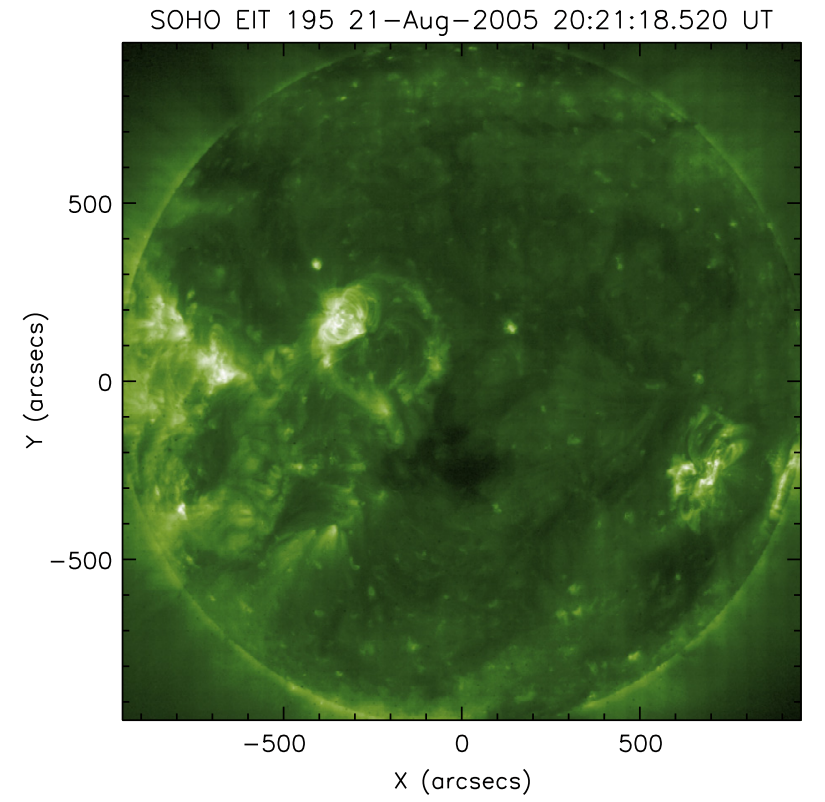

Fig. 1. SOHO/EIT $195 \AA$ image showing two solar anemones, $A R$ 10798 (centered around coordinates $[700,-300]$ ) and AR 10800 (circular shaped, located around coord. [200, 100], in arcsecs).

X17.2 class) and another one from this AR on 29 October at 20:54 UT, where AR 10488 behaved sympathetically, and the associated flare was X10.0. The solar magnetic cause might be the reconnection of counter-helical flux ropes (Liu et al. 2007). The event is fully described in Liu et al. (2006). The plane-of-sky speed of the CMEs ranged from 1400 to $2500 \mathrm{~km} \mathrm{~s}^{-1}$. All flares happened $\sim 30 \mathrm{~min}$ before the CME was identified by LASCO. These CMEs were modelled as flux ropes by Krall et al. (2006), also analysing the magnetospheric response.

The superflare 4B/X17.2 on 28 October (and re-classified as even larger, $\mathrm{X} 45$ ) was registered on the very complex active region NOAA 10486. Different features were recorded, such as white-light flaring, gamma-ray emission, $\mathrm{KeV}$ particle emission and Moreton waves (Hurford et al. 2006; Maurya \& Ambastha 2009; Muhr et al. 2010). The magnetic topology of this region is very intrincate, as emerging bipoles were coalescing to form a very complex region with a coronal sheared arcade. A new emerging bipole appeared close to the flaring spot in the quadrupolar region, as investigated in Schmieder et al. (2006). The violent dynamics has been described in del Zanna et al. (2006). The magnetic structure has been analysed also in Su et al. (2006); Dun et al. (2007), among others.

The geomagnetic storm of 24 August 2005 is an unusual event, and not so evident as the previous cases. These CMEs were ejected on 22 August at 1:31 UT and 17:30 UT, each one related to M2.6 and M5.6 flares. The plane-of-the-sky speeds were also high, around 1200 and $2400 \mathrm{~km} \mathrm{~s}^{-1}$ respectively. Close to an equatorial coronal hole, a large dimming was produced after the $\mathrm{CME}$, and even increased its area with the second CME (Cerrato et al. 2012). This dimming resulted in a larger $\mathrm{CH}$, which was seen as a Corotating Interaction Region (CIR) at interplanetary medium.

The active region source of these two CMEs was AR 10798 (as shown in Fig. 1), an anemone region - an active region that grew into a $\mathrm{CH}$ - with the singularity of being polarity reversed compared to the preceding sunspot, and without following Hale's law. The fountain-like shape is due to the 
active region reconnection with the coronal hole mainly unipolar magnetic field (Asai et al. 2008, and references therein). The ejected filament was analysed thoroughly in e.g. Asai et al. (2009). Lugaz et al. (2011) investigated the case via observational data and numerical simulations, and concluded that the flux rope expanded and was deflected by the coronal hole. Actually, coronal holes may deflect CMEs (Lugaz et al. 2011; Kahler et al. 2012). As we have indicated, CHs played an important role on the 24 August 2005 storm's case.

However, in other geomagnetic storm examples, these solar triggers and their circumstances can be much more elusive. Since more than $80 \%$ of CMEs are not associated with a large flare (Linker et al. 2003), taking flares as the only precursors of geoeffectiveness, without considering other features may be insufficient. Also, cases of flare-less CMEs were investigated in Song et al. (2013). The position of the AR in the solar disc is not definite either for being geoeffective: an eruptive active region located in the central meridian is easy to identify as main precursor, but active regions can be also geoeffective when located close to the limb (Cid et al. 2012). Besides, there are very distinct cases of Earth-directed CMEs, called "stealth CMEs" (e.g., Webb \& Howard 2012), where automated detection methods fail and only a detailed study can find them. On the other hand, recognising these phenomena in the interplanetary medium may be also complicated, due to lack of clarity in the solar source identification, or structure interactions in the interplanetary medium, e.g., shocks without apparent drivers as in Gopalswamy et al. (2009).

\section{On the main phase of extreme storms: from Dst index to local magnetometer records}

Most problems in utilities have been related to the main phase of the geomagnetic storm (when the decrease of the Dst index takes place) or during the sudden commencement (which appears as a Dst increase at the beginning of the storm) since during these phases some magnetospheric currents are enhanced and the terrestrial magnetic field varies (see e.g., Ngwira et al. 2013b). The Dst index, which was introduced as a measurement of the ring current encircling the Earth (Dessler \& Parker 1959; Sckopke 1966), is considered as a good estimation of the geomagnetic disturbance at mid-low latitudes. As a global index, the Dst index is obtained by averaging records from several magnetometers located at mid-low latitudes spread in longitude.

In some cases, Dst is not available, but it can be reconstructed in a similar way. Following the method by Cid et al. (2013), similar to Dst computation procedure, we define an index named $D s t^{\prime}$ by averaging magnetic disturbances at different low-latitude observatories, as follows:

$$
D s t^{\prime}=\frac{1}{N} \sum_{i=1}^{N} \text { Disturbance }_{i},
$$

where

$$
\text { Disturbance }_{i}=\frac{H(t)_{i}-H_{\text {baseline }, i}}{\cos \phi_{i}} .
$$

$N$ corresponds to the number of magnetic observatories involved in computing $D s t^{\prime}, i$ represents each observatory located at a magnetic latitude $\phi, H$ is the horizontal magnetic
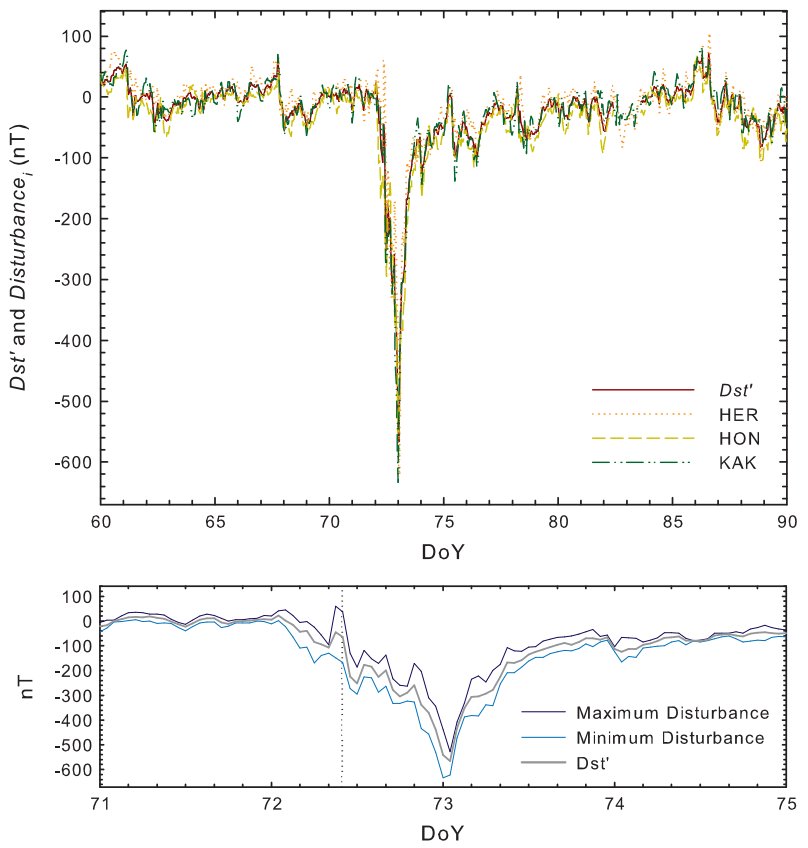

Fig. 2. Top: $D s t^{\prime}$ index and local disturbance recorded at the observatories of Hermanus, Kakioka, Honolulu for the 13 March 1989 storm. There are no data from San Juan magnetometer. Bottom: Dst' index (grey) and the local time maximum (dark blue) and minimum (light blue) value of the Disturbance ${ }_{i}$.

component, $H_{\text {baseline }}$ is the $H$-component quiet-day model, as defined in Cid et al. (2013).

The observatory $i$ is expected to experience a Disturbance ${ }_{i}$ (Eq. (2)) which will be similar to each other only if the magnetic disturbance is associated to an approximately symmetric ring current. This statement is far to be true during the main phase of severe storms.

We have computed the Disturbance ${ }_{i}$ for the extreme geomagnetic storm in March 1989, from the observatory records of Hermanus (HER), Kakioka (KAK) and Honolulu (HON) (there are not measurements available from San Juan (SJG) observatory), and then, $D s t^{\prime}$ (Eq. (1)). Disturbance ${ }_{i}$ and $D s t^{\prime}$ are shown in the top panel in Figure 2. Dst' is very similar to the Dst provided by Kyoto.

Even though Disturbance ${ }_{i}$ from every observatory seems to be similar to each other and to Dst, as displayed in the top panel in Figure 2, differences between disturbances measured at different observatories reached more than $200 \mathrm{nT}$ at some moments. The bottom panel in Figure 2 displays the Dst and the local time maximum and minimum value of the Disturbance $_{i}$. The disturbance is clearly asymmetric during the long (almost one day) main phase of the storm and during the early recovery phase. Soon after starting the decrease of the Dst, when Dst $=-44 \mathrm{nT}$, the asymmetry grew up to $208 \mathrm{nT}$. The large deviation from Dst at this time is mainly due to the disturbance recorded at HER at about 09:00 LT (vertical dashed line).

Although quantitatively different, Disturbance ${ }_{i}$ profiles are qualitatively similar in March 1989 storm. However, this is not a general case. For instance, the severe storm on 29 October 2003 looks quite different at low-latitude observatories, as HER and SJG, usually involved in the Dst computation. These differences in profile shape and intensity are even larger 

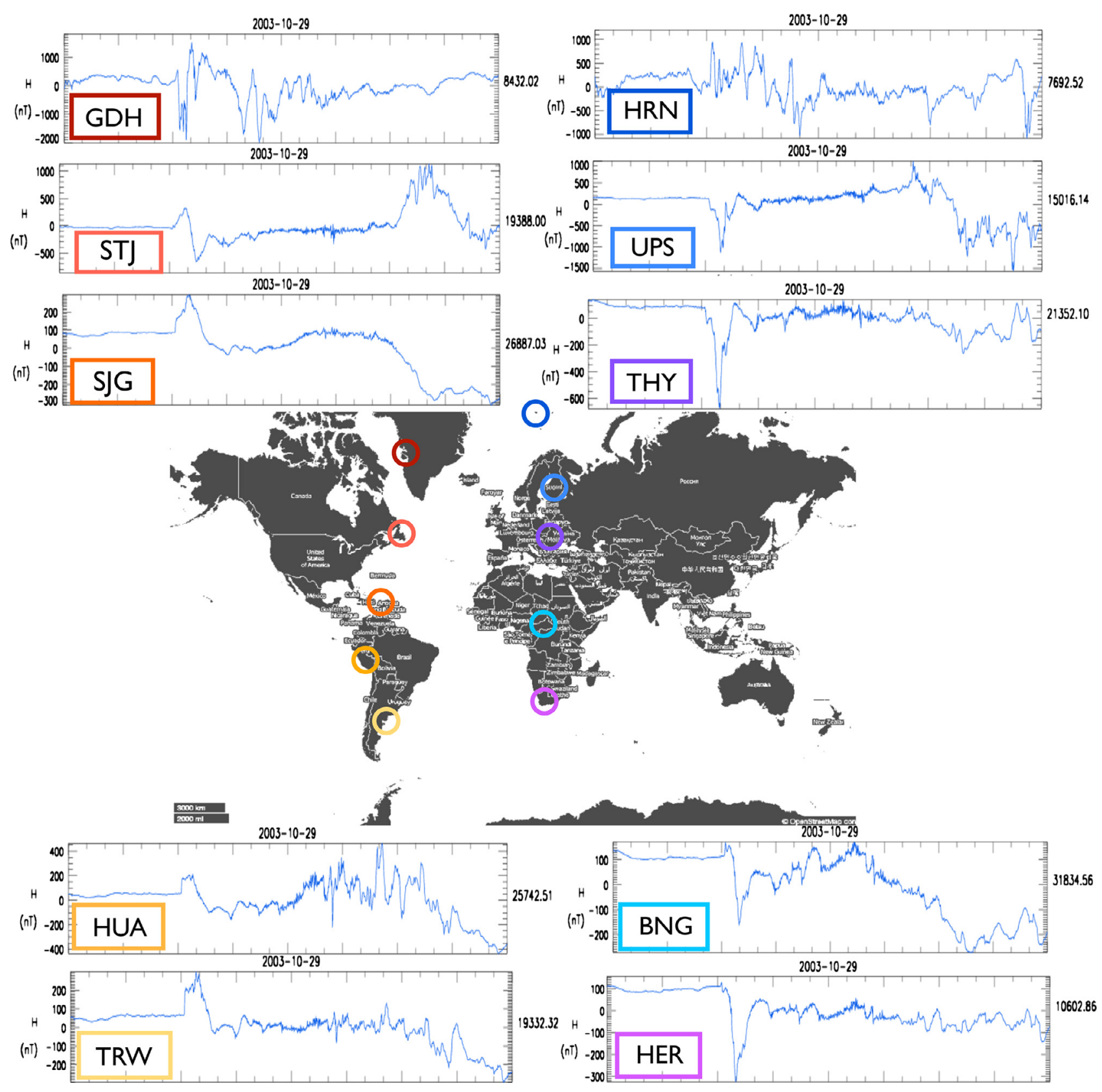

Fig. 3. Map showing $H$ records along two main longitudes: $300^{\circ}$ and $10^{\circ}$. The profiles of the $H$ component are clearly different at different locations on 29 October 2003. Offset values are shown at the right of each panel.

considering a variety of longitudes. Figure 3 shows the horizontal component $H$ during the 29 October 2003 (the first day of the Halloween storm) recorded at ten magnetometers. An offset value corresponding to the average of the horizontal component $H$ recorded during the previous day (shown on the right vertical axis in the Figure) has been considered in the scale in order to estimate the disturbance. The observatories have been selected in order to cover high, middle and low latitudes at both hemispheres for two different longitude ranges. Thus, HRN, UPS, THY, BNG and HER are located at a geographical longitude of $10 \pm 10^{\circ}$ (circles in red hue in Fig. 3 show exact location) and GDH, STJ, SJG, HUA and TRW at $300 \pm 15^{\circ}$ (circles in blue shade are used in this case). Just a first glance is needed to appreciate the large deviation from one to another local disturbance recorded at different locations.
Hence, observatories in Northern hemisphere at longitudes close to $300^{\circ}$ show a large disturbance at high latitude (of about $2000 \mathrm{nT}$ ), which diminishes when latitude decreases (Watermann \& Gleisner 2009). The disturbance in the Southern hemisphere is significantly smaller than in the Northern one for any latitude considered. However, at longitudes close to $10^{\circ}$, the disturbances in the Northern hemisphere do not decrease from high to low latitudes, resulting in an extreme disturbance of almost $800 \mathrm{nT}$ at Tihany (THY, geographic latitude $45^{\circ} 99$ ). The northern-southern asymmetry remains at this longitude.

The above examples evidence that global indices are not adequate to quantify the geomagnetic disturbance according to its hazard. They also raise some important concerns regarding the existence and relevance of local geomagnetic indices. 
Besides the local or global character of the index, its temporal resolution is also an important issue. $K$-indices are the typical approach to local indices (e.g., Nowożyński et al. 1991). However, $K$-indices have 3 -h resolution, which is not enough to monitor fast magnetic field variations that are involved in GICs, which are major effects of the severe geomagnetic disturbances with consequences to society. Higher temporal resolution local indices are needed for an efficient societal assessment of space weather.

\section{On the recovery phase: the not-so-unique Carrington recovery phase}

Cid et al. (2013) analysed the recovery phase of the "largest magnetic storms" occurred since 1857 from the Table 1 of Tsurutani et al. (2003). Unable to reproduce the recovery of these magnetic records by an exponential function, these authors checked whether the events listed from low latitude stations comply with the function proposed by Aguado et al. (2010). This function, which proposed a hyperbolic decay for the recovery phase of Dst for intense storms, is given by the following expression:

$$
D s t(t)=\frac{D s t_{0}}{1+\frac{t}{\tau_{h}}},
$$

being $D s t_{0}$ the minimum value of $D s t$ index reached at $t=0$ (when the recovery phase begins), and $\tau_{h}$ the characteristic recovery time. The recovery time of the hyperbolic function, $\tau_{h}$, represents the time needed to reach the half of the initial value (initial value as $D s t_{0}$ ). An empirical relationship was also proposed for the recovery time as proportional to the intensity of the storm (quantified by $D s t_{0}$ ). In this way, the hyperbolic decay function provides two major advantages when comparing with the exponential one: (1) it better fits experimental data, considering the dependence of the recovery time on the intensity, and (2) it results in a non-linear dependence of the $\mathrm{d} D s t / \mathrm{d} t$ upon Dst, which is consistent with several loss processes involved at different stages in the recovery phase of the magnetospheric current system.

The worth of the hyperbolic model for reproducing those experimental data lies in the physics underneath the hyperbolic function. This mathematical function reproduces those physical processes where there is a non-constant degree of reduction of a magnitude and, as a consequence, a non-linear coupling of the rate of change of the magnitude relative to itself. Examples of that behaviour appear in several disciplines. The learning process of a foreign language is an example of this hyperbolic behaviour: at the beginning, the student learns a lot every day, but after some time learning the language, a light improvement in skills requires a large effort. In the same way, the losses of energy in the magnetosphere might not be proportional to the energy content itself, as indicated by an exponential decay, but proportional to the square of the energy content, as indicated by the hyperbolic function.

The hyperbolic function was proposed initially for the Dst index, but this index was not available for the largest magnetic storms ever recorded. Therefore Cid et al. (2013) estimated the Dst index from what they called the $L D i$ (Local Disturbance index). This index included not only the disturbance of $H$ at an specific station but also two corrections to take into account: (1) the magnetic latitude of the observatory to normalize the index to the dipole equator, and (2) the local time of the magnetometer. This last correction is the main difference between $L D i$ and Disturbance ${ }_{i}$ as defined above.

The results obtained by Cid et al. (2013) revealed the high accuracy of the hyperbolic decay function to reproduce the recovery phase after an extreme storm from local magnetic records. However, they also evidenced that the relationship between the two parameters involved in the hyperbolic decay ( $\tau_{h}$ and $D s t_{0}$ ) was not a linear function, suggesting an exponential trend. This function was proposed mainly forced by the small recovery time obtained for the Carrington storm, $8.4 \mathrm{~min}$, completely different from the other events analysed in that work, which ranged from 3.5 to $8.4 \mathrm{~h}$. Thus, Carrington storm was not only unique because its large disturbance recorded at Colaba observatory, but also because its very fast recovery, which was noticed as a key factor for theoretical modelling (Li et al. 2006). Nevertheless, this exceptional feature might also be related to the fact that local magnetic records were considered in the analysis.

Other examples of this less-than-one-hour recovery phase can be discovered just by checking individual magnetic records of extreme disturbances, namely: 16 April 1938 in Niemegk (NGK), 14 March 1989 in Borok (BOX) and 29 October 2003 in Tihany (THY).

In Figure 4, top left panel shows $L D i$ for Colaba during the Carrington event and other three events mentioned above at local observatories. Here $L D i$ has not included the latitude and local-time corrections, because the aim in this case is to study local disturbances and not to obtain a global index (like $D s t$ or $D s t^{\prime}$ indices) neither to compare disturbances at different latitudes (by cosine correction) or longitudes (by local-time correction).

During the event on 16 April 1938 (top right panel) at NGK, the hourly resolution $L D i$ at that station recovered from $-646 \mathrm{nT}$ at $7 \mathrm{UT}$ to $-43 \mathrm{nT}$ at $8 \mathrm{UT}$, decaying again like a two-step storm. More recently, during 13 March 1989, other magnetometers at mid-high latitudes such as BOX recorded larger disturbances than that of KAK. Calculated $L D i$ for BOX (bottom left panel) registered a peak value of $-1684 \mathrm{nT}$. Then, it recovered almost $1000 \mathrm{nT}$ in $1 \mathrm{~h}$, diminishing again to $-1075 \mathrm{nT}$, developing a double-step storm. Finally, quiet time values are recovered $5 \mathrm{~h}$ after the largest disturbance. Also some local magnetic records for the October 2003 storm (see Fig. 3) show a similar recovery phase. Specifically, THY magnetic records (bottom right panel in Fig. 4) show undisturbed values $45 \mathrm{~min}$ after the maximum disturbance $(L D i=-777 \mathrm{nT})$, extraordinarily resembling the Carrington storm.

The hyperbolic function is used to fit $L D i$ for cases presented in Figure 5. The fitting results for these events are listed in Table 1. For the case of 1938, only hourly resolution data are available and therefore no fitting can be performed since the recovery phase only lasted $1 \mathrm{~h}$. When a double peak appeared in $L D i$ data, two fittings are performed for different temporal intervals, one until the beginning of the second peak, and another one until an additional significant drop appears. If no significant drop is observed, a 48-h interval is used following the criterium in Aguado et al. (2010). The fitting time interval $(\Delta t)$ is shown in column 2 in Table 1 . For the event of 1989 the double peak starts $3 \mathrm{~h}$ after the $L D i$ peak, therefore, a fitting with only three experimental points has not significance. A correlation coefficient $R$, always above 0.82 , indicates the goodness of the hyperbolic function. Nevertheless, it can be noticed that different time intervals do not modify considerably 
C. Cid et al.: On extreme geomagnetic storms
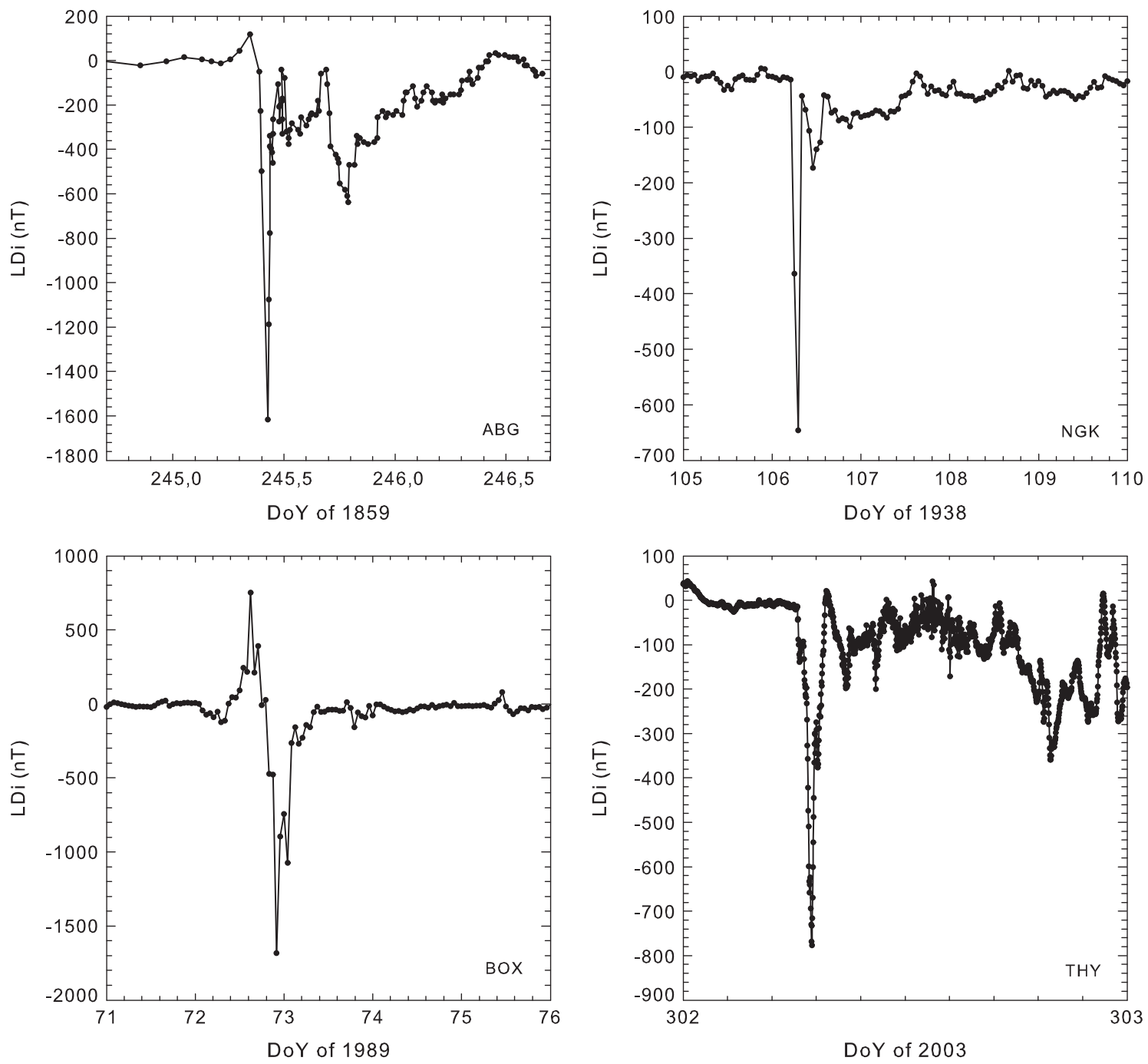

Fig. 4. The computed $L D i$ for the events on September 1859 at ABG, April 1938 at NGK, March 1989 at BOX and October 2003 at THY, from left to right and top to bottom.
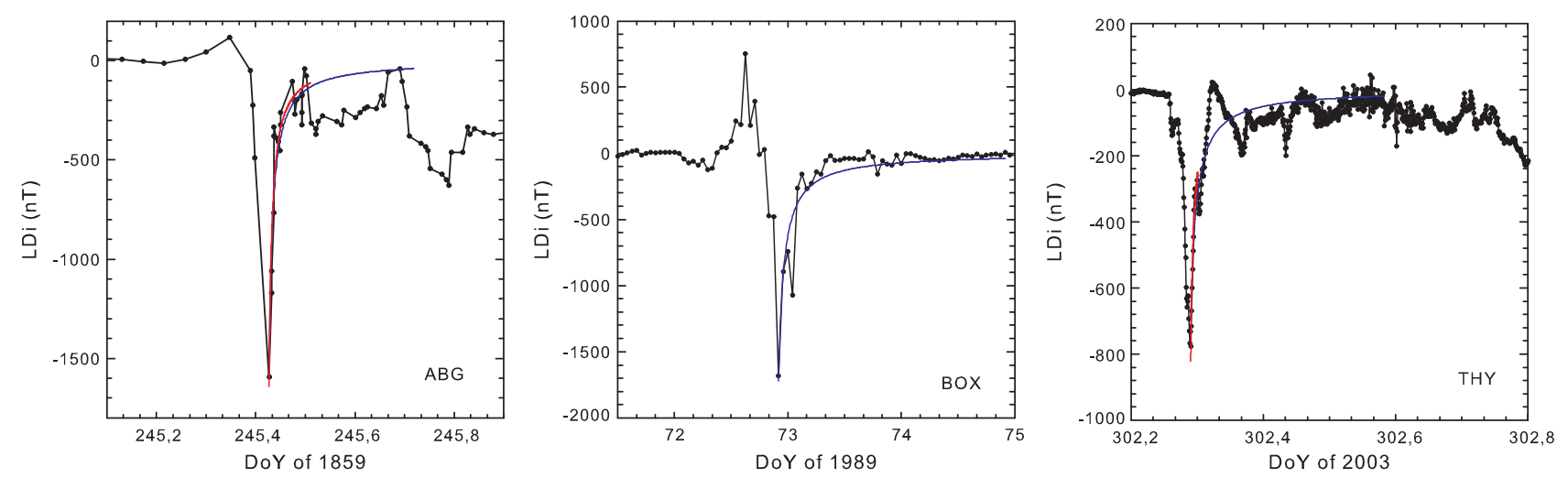

Fig. 5. Fitting results superposed to $L D i$ for those analysed events (ABG, BOX, THY). Different colours indicate different time intervals in the fitting procedure: \#1 (\#2) results in Table 1 corresponds to red (blue) solid line.

the final results of the fitting as the most significant feature in the recovery is the impulsive rise in the beginning, which is only reproduced by a hyperbolic decay function.

A preliminary interpretation of the results in Table 1 might suggest that the parameters $L D i_{0}$ and $\tau_{h}$ obtained for these three events do not follow the trend proposed by Cid et al. (2013), as $\tau_{h}$ does not decrease as the peak in $L D i$ increases. However, this is misleading, since the parameters from both studies are not comparable. The explanation for this last statement is based on the correct understanding of the hyperbolic model. This model provides a unique continuous function to address the existence of diverse processes of different nature (flow-out, charge exchange 
Table 1. This table presents several parameters of the hyperbolic fitting for the events.

\begin{tabular}{lcccc}
\hline \hline Event & $\Delta t_{\text {fitting }}[\mathrm{h}]$ & $L D i_{0}[\mathrm{nT}]$ & $\tau_{h}[\mathrm{~h}]$ \\
\hline 1859 ABG \#1 & 1.7 & $-1640 \pm 140$ & $0.15 \pm 0.03$ & $0.18 \pm 0.04$ \\
1859 ABG \#2 & 6.33 & $-1560 \pm 160$ & $1.14 \pm 0.16$ \\
1989 BOX & 48 & $-1700 \pm 100$ & $0.124 \pm 0.018$ \\
2003 THY \#1 & 0.93 & $-820 \pm 60$ & $0.208 \pm 0.016$ & 0.85 \\
2003 THY \#2 & 7 & $-720 \pm 30$ & 0.91 \\
\hline
\end{tabular}

Notes. $\# 1$ and \#2 correspond to different fittings of the same events considering different time intervals.

of different ions, particle precipitation by wave-particle interaction, etc.) through an empirical parameter: $\tau_{h}$, which depends on the intensity peak, $L D i_{0}$. But $\tau_{h}$ is far from being a unique and constant value - for a given $L D i_{0}$ - able to reproduce the recovery of magnetic disturbances at different latitudes, as that of BOX, THY or ABG. A comparison between hyperbolic model and a well-known empirical model as Hooke's law can help to understand the previous statement.

It is an experimental fact that, under some specific conditions, the strain of elastic objects is proportional to the stress applied to it. Although Hooke's law holds - as a first approach - to any elastic object, the "proportionality factor" will remain unchanged while keeping the same material. Otherwise, molecular forces will change and as a result the proportionality factor will change. In an equivalent way, we have shown in this paper that hyperbolic model is able to reproduce the recovery phase after a severe disturbance whatever will be the place (high, middle or low latitudes) where the recovery phase is measured. However, if location is changed, the currents involved and the physical processes involved in the decay will also change. As a result, the "constant" of the hyperbolic model $\left(\tau_{h}\right)$ cannot be considered as an unchanging value for a given intensity peak and any magnetospheric region and therefore it may change, at least with latitude.

\section{Conclusions}

Extreme storms are very unusual phenomena and therefore collecting reports of these events is extremely difficult. As a consequence, these extreme storms are not statistically significant when analysing disturbed geomagnetic conditions. This fact might prevent us to extrapolate severe storms from moderate or intense ones. Further detailed case studies may clarify the issue.

In the Introduction section, we present by four questions the key-issues related to extreme space weather that, in our opinion, need to be carefully addressed by scientific community. After analysing in this paper some available events, trying to provide an answer to those questions, the main conclusions of this study are the following:

1. CME chains and interaction between different structures, including fast streams from coronal holes, are involved in the events analysed, in agreement with previous results (Wang et al. 2003; Xie et al. 2006; Zhang et al. 2007; Cid et al. 2008; Yermolaev \& Yermolaev 2008). Nevertheless, not all CME chains and interactions lead to extreme geomagnetic storms with reported damages (the case of 24 August 2005 is an example). Solar features such as large shear, flux emergence and cancellation and large transient coronal dimmings appear as common features in these largest events. Also all three events present large solar wind speed, as expected. Major efforts should be dedicated to this issue in future works.

2. Local magnetic disturbances seem to play a key role in assessing the potential risk factor of extreme events in specific regions. Therefore, the term "extreme storm" should not be associated with any threshold of any global geomagnetic index, as Dst $<-250 \mathrm{nT}$. Indices such as the $L D i$, based in the proposal by Cid et al. (2013), but without latitude correction, as done in this paper, compute one-minute resolution local disturbances from the records of a specific magnetometer; hence, they shall provide a valuable contribution, as they will constitute both a well defined estimator of the disturbance according to scientific standards and a useful tool for the users community for future studies on effect-based scales. A high resolution local scale based in indices as $L D i$ and related to technological effects should be established to redefine the term "extreme storm", or even better, to define the term extreme geomagnetic disturbance. Collaboration between scientific community and users community is necessary for this issue.

3. The influence of latitude in geomagnetic storms is well known, as geomagnetic disturbances are usually larger at high latitudes due to the effect of auroral electrojet. Although some previous studies mentioned a longitude dependence of magnetic records (Tyasto et al. 2009; Love \& Gannon 2010), in this study longitude appears as a relevant factor for the severity of the disturbance.

4. The recovery of local disturbances after a extreme disturbance follows a hyperbolic law, whatever the latitude considered, indicating that the losses of energy in the magnetosphere depends quadratically on its own energy content. Additional efforts should be dedicated to obtained $\tau_{h}$ values for different latitudes and intensities in order to determine relationships between parameters.

Acknowledgements. We acknowledge data from the World Data Center for Geomagnetism, Kyoto (WDC), and the national institutions that support the magnetic observatories providing data for INTERMAGNET. Also EIT, MDI and LASCO data from SOHO, and LASCO catalogue are acknowledged. Map credits of GeoFabrik and (C) OpenStreetMap contributors.

The editor thanks two anonymous referees for their assistance in evaluating this paper.

\section{References}

Aguado, J., C. Cid, E. Saiz, and Y. Cerrato, Hyperbolic decay of the Dst index during the recovery phase of intense geomagnetic storms, J. Geophys. Res. [Space Phys.], 115, A07220, 2010. 
Asai, A., K. Shibata, H. Hara, and N.V. Nitta, Characteristics of anemone active regions appearing in coronal holes observed with the Yohkoh soft X-ray telescope, ApJ, 673, 1188-1193, 2008.

Asai, A., K. Shibata, T.T. Ishii, M. Oka, R. Kataoka, K. Fujiki, and N. Gopalswamy, Evolution of the anemone AR NOAA 10798 and the related geo-effective flares and CMEs, J. Geophys. Res. [Space Phys.], 114, A00A21, 2009.

Aulanier, G., The physical mechanisms that initiate and drive solar eruptions, IAU Symposium, 300, 184-196, 2014.

Baker, D.N., X. Li, A. Pulkkinen, C.M. Ngwira, M.L. Mays, A.B. Galvin, and K.D.C. Simunac, A major solar eruptive event in July 2012: defining extreme space weather scenarios, Space Weather, 11, 585-591, 2013.

Carrington, R.C., Description of a singular appearance seen in the Sun on September 1, 1859, MNRAS, 20, 13-15, 1859.

Cerrato, Y., E. Saiz, C. Cid, W.D. Gonzalez, and J. Palacios, Solar and interplanetary triggers of the largest Dst variations of the solar cycle 23, J. Atmos. Sol. Terr. Phys., 80, 111-123, 2012.

Cid, C., H. Cremades, A. Aran, C. Mandrini, B. Sanahuja, et al., Can a halo CME from the limb be geoeffective? J. Geophys. Res. [Space Phys.], 117, A11102, 2012.

Cid, C., J. Palacios, E. Saiz, Y. Cerrato, J. Aguado, and A. Guerrero, Modeling the recovery phase of extreme geomagnetic storms, J. Geophys. Res. [Space Phys.], 118, 4352-4359, 2013.

Cid, C., E. Saiz, and Y. Cerrato, Comment on "Interplanetary conditions leading to superintense geomagnetic storms (Dst $\leq=-250 \mathrm{nT}$ ) during solar cycle 23 " by E. Echer et al., Geophys. Res. Lett., 35, L21107, 2008.

del Zanna, G., B. Schmieder, H. Mason, A. Berlicki, and S. Bradshaw, The gradual phase of the X17 Flare on October 28, 2003, Sol. Phys., 239, 173-191, 2006.

Démoulin, P., C.H. Mandrini, L. van Driel-Gesztelyi, B.J. Thompson, S. Plunkett, Z. Kovári, G. Aulanier, and A. Young, What is the source of the magnetic helicity shed by CMEs? The long-term helicity budget of AR 7978, $A \& A, 382,650-665$, 2002.

Deng, Y., J. Wang, Y. Yan, and J. Zhang, Evolution of magnetic nonpotentiality in NOAA AR 9077, Sol. Phys., 204, 11-26, 2001.

Dessler, A.J., and E.N. Parker, Hydromagnetic theory of geomagnetic storms, J. Geophys. Res., 64 (12), 2239-2252, DOI: 10.1029/JZ064i012p02239, 1959.

Dun, J., H. Kurokawa, T.T. Ishii, Y. Liu, and H. Zhang, Evolution of magnetic nonpotentiality in NOAA AR 10486, ApJ, 657, 577-591, 2007.

Echer, E., W.D. Gonzalez, and B.T. Tsurutani, Interplanetary conditions leading to superintense geomagnetic storms (Dst $\leq-250 \mathrm{nT}$ ) during solar cycle 23, Geophys. Res. Lett., 35, L06S03, 2008.

Gaunt, C., and G. Coetzee, Transformer failures in regions incorrectly considered to have low gic-risk, in: Power Tech, 2007 IEEE Lausanne, pp. 807-812, 2007.

Gonzalez, W.D., E. Echer, A.L. Clúa de Gonzalez, B.T. Tsurutani, and G.S. Lakhina, Extreme geomagnetic storms, recent Gleissberg cycles and space era-superintense storms, J. Atmos. Sol. Terr. Phys., 73, 1447-1453, 2011.

Gonzalez, W.D., E. Echer, A.L. Clua-Gonzalez, and B.T. Tsurutani, Interplanetary origin of intense geomagnetic storms (Dst $\leq-100$ nT) during solar cycle 23, Geophys. Res. Lett., 34, L06101, 2007.

Gonzalez, W.D., J.A. Joselyn, Y. Kamide, H.W. Kroehl, G. Rostoker, B.T. Tsurutani, and V.M. Vasyliunas, What is a geomagnetic storm? J. Geophys. Res., 99, 5771-5792, 1994.

Gopalswamy, N., P. Mäkelä, H. Xie, S. Akiyama, and S. Yashiro, CME interactions with coronal holes and their interplanetary consequences, J. Geophys. Res. [Space Phys.], 114, A00A22, 2009

Gosling, J.T., The solar flare myth, J. Geophys. Res., 98 (A11), 18937-18949, DOI: 10.1029/93JA01896, 1993.

Hurford, G.J., S. Krucker, R.P. Lin, R.A. Schwartz, G.H. Share, and D.M. Smith, Gamma-ray imaging of the 2003 October/November solar flares, ApJ, 644, L93-L96, 2006.
Jing, J., V.B. Yurchyshyn, G. Yang, Y. Xu, and H. Wang, On the relation between filament eruptions, flares, and coronal mass ejections, ApJ, 614, 1054-1062, 2004.

Kahler, S.W., S. Akiyama, and N. Gopalswamy, Deflections of fast coronal mass ejections and the properties of associated solar energetic particle events, ApJ, 754, 100, 2012.

Kataoka, R., Probability of occurrence of extreme magnetic storms, Space Weather, 11, 214-218, 2013.

Krall, J., V.B. Yurchyshyn, S. Slinker, R.M. Skoug, and J. Chen, Flux rope model of the 2003 October 28-30 coronal mass ejection and interplanetary coronal mass ejection, ApJ, 642, 541-553, 2006

Kusano, K., Y. Bamba, T.T. Yamamoto, Y. Iida, S. Toriumi, and A. Asai, Magnetic field structures triggering solar flares and coronal mass ejections, ApJ, 760, 31, 2012.

Lakhina, G.S., S. Alex, B.T. Tsurutani, and W.D. Gonzalez, Research on historical records of geomagnetic storms. In: K., Dere, J. Wang, and Y. Yan, Editors, Coronal and Stellar Mass Ejections, vol. 226 of IAU Symposium, pp. 3-15, 2005.

Li, X., M. Temerin, B.T. Tsurutani, and S. Alex, Modeling of 1-2 September 1859 super magnetic storm, Adv. Space Res., 38, 273-279, 2006

Linker, J.A., Z. Mikić, P. Riley, R. Lionello, and D. Odstrcil, Models of coronal mass ejections: a review with a look to the future. In: M., Velli, R. Bruno, F. Malara, and B. Bucci, Editors, Solar Wind Ten, vol. 679 of American Institute of Physics Conference Series, pp. 703-710, 2003.

Liu, C., J. Lee, N. Deng, D.E. Gary, and H. Wang, Large-scale activities associated with the 2003 October 29 X10 flare, ApJ, 642, 1205-1215, 2006.

Liu, Y., H. Kurokawa, C. Liu, D.H. Brooks, J. Dun, T.T. Ishii, and H. Zhang, The X10 flare on 29 October 2003: Was it triggered by magnetic reconnection between counter-helical fluxes? Sol. Phys., 240, 253-262, 2007.

Liu, Y.D., J.G. Luhmann, P. Kajdič, E.K.J. Kilpua, N. Lugaz, et al., Observations of an extreme storm in interplanetary space caused by successive coronal mass ejections, Nature Communications, $\mathbf{5}$, 3481, 2014.

Love, J.J., and J.L. Gannon, Movie-maps of low-latitude magnetic storm disturbance, Space Weather, 8, 06001, 2010.

Lugaz, N., C. Downs, K. Shibata, I.I. Roussev, A. Asai, and T.I. Gombosi, Numerical investigation of a coronal mass ejection from an anemone active region: reconnection and deflection of the 2005 August 22 eruption, ApJ, 738, 127, 2011.

Maurya, R.A., and A. Ambastha, Transient magnetic and doppler features related to the white-light flares in NOAA 10486, Sol. Phys., 258, 31-52, 2009.

Muhr, N., B. Vršnak, M. Temmer, A.M. Veronig, and J. Magdalenić, Analysis of a global Moreton wave observed on 2003 October 28, ApJ, 708, 1639-1649, 2010.

Ngwira, C.M., A. Pulkkinen, M.M. Kuznetsova, and A. Glocer, Modeling extreme carrington-type' space weather events using three-dimensional global mhd simulations, J. Geophys. Res. [Space Phys.], 119 (6), 4456-4474, DOI: $10.1002 / 2013$ JA019661, 2014

Ngwira, C.M., A. Pulkkinen, M. Leila Mays, M.M. Kuznetsova, A.B. Galvin, et al., Simulation of the 23 July 2012 extreme space weather event: What if this extremely rare CME was Earth directed? Space Weather, 11, 671-679, 2013a.

Ngwira, C.M., A. Pulkkinen, F.D. Wilder, and G. Crowley, Extended study of extreme geoelectric field event scenarios for geomagnetically induced current applications, Space Weather, 11, 121-131, 2013b.

Nowożyński, K., T. Ernst, and J. Jankowski, Adaptive smoothing method for computer derivation of K-Indices, Geophys. J. Int., 104, 85-93, 1991 .

Rodriguez, L., A.N. Zhukov, C. Cid, Y. Cerrato, E. Saiz, et al., Three frontside full halo coronal mass ejections with a nontypical geomagnetic response, Space Weather, 7, S06003, 2009. 
Russell, C.T., R.A. Mewaldt, J.G. Luhmann, G.M. Mason, T.T. von Rosenvinge, et al., The very unusual interplanetary coronal mass ejection of 2012 July 23: a blast wave mediated by solar energetic particles, ApJ, 770, 38, 2013.

Schmieder, B., C.H. Mandrini, P. Démoulin, E. Pariat, A. Berlicki, and E. Deluca, Magnetic reconfiguration before the X 17 solar flare of October 28 2003, Adv. Space Res., 37, 1313-1316, 2006.

Sckopke, N., A general relation between the energy of trapped particles and the disturbance field near the Earth, J. Geophys. Res., 71, 3125-3130, 1966.

Siscoe, G.L., A quasi-self-consistent axially symmetric model for the growth of a ring current through earthward motion from a pre-storm configuration, Planet. Space Sci., 27, 285-295, 1979.

Song, H.Q., Y. Chen, D.D. Ye, G.Q. Han, G.H. Du, G. Li, J. Zhang, and Q. Hu, A study of fast flareless coronal mass ejections, ApJ, 773, 129, 2013

Srivastava, N., Predicting the occurrence of super-storms, Ann. Geophys., 23, 2989-2995, 2005.

$\mathrm{Su}$, Y.N., L. Golub, A.A. van Ballegooijen, and M. Gros, Analysis of magnetic shear in an X17 solar flare on October 28, 2003, Sol. Phys., 236, 325-349, 2006.

Svestka, Z., and E.W. Cliver, History and basic characteristics of eruptive flares. In: Z., Svestka, B.V. Jackson, and M.E. Machado, Editors, IAU Colloq. 133: Eruptive Solar Flares, vol. 399 of Lecture Notes in Physics, Berlin Springer Verlag, p.1, 1992.

Thomson, A.W.P., C.T. Gaunt, P. Cilliers, J.A. Wild, B. Opperman, L.-A. McKinnell, P. Kotze, C.M. Ngwira, and S.I. Lotz, Present day challenges in understanding the geomagnetic hazard to national power grids, Adv. Space Res., 45, 1182-1190, 2010.

Tsurutani, B.T., W.D. Gonzalez, G.S. Lakhina, and S. Alex, The extreme magnetic storm of 1-2 September 1859, J. Geophys. Res. [Space Phys.], 108, 1268, 2003.
Tyasto, M.I., N.G. Ptitsyna, I.S. Veselovsky, and O.S. Yakovchouk, Extremely strong geomagnetic storm of September 2-3, 1859, according to the archived data of observations at the Russian network, Geomag. Aeron., 49, 153-162, 2009.

van Ballegooijen, A.A., and P.C.H. Martens, Formation and eruption of solar prominences, ApJ, 343, 971-984, 1989.

Wang, Y.M., P.Z. Ye, and S. Wang, Multiple magnetic clouds: several examples during March-April 2001, J. Geophys. Res. [Space Phys], 108, 1370, 2003.

Watermann, J., and H. Gleisner, Geomagnetic variations and their time derivatives during geomagnetic storms at different levels of intensity, Acta Geophys., 57, 197-208, 2009.

Webb, D.F., and T.A. Howard, Coronal mass ejections: observations, Living Rev. Sol. Phys., 9, 3, 2012.

Xie, H., N. Gopalswamy, P.K. Manoharan, A. Lara, S. Yashiro, and S. Lepri, Long-lived geomagnetic storms and coronal mass ejections, J. Geophys. Res. [Space Phys.], 111, A01103, 2006.

Yermolaev, Y.I., I.G. Lodkina, N.S. Nikolaeva, and M.Y. Yermolaev, Occurrence rate of extreme magnetic storms, J. Geophys. Res. [Space Phys.], 118, 4760-4765, 2013.

Yermolaev, Y.I., and M.Y. Yermolaev, Comment on "Interplanetary origin of intense geomagnetic storms (Dst $\leq-100 \mathrm{nT}$ ) during solar cycle 23" by W. D. Gonzalez et al., Geophys. Res. Lett., 35, L01101, 2008.

Yizengaw, E., P. Doherty, and T. Fuller-Rowell, Is space weather different over Africa, and if so, why? An agu chapman conference report, Space Weather, 11 (7), 389-391, DOI: 10.1002/swe.20063, 2013.

Zhang, J., I.G. Richardson, D.F. Webb, N. Gopalswamy, E. Huttunen, et al., Solar and interplanetary sources of major geomagnetic storms (Dst $\leq-100 \mathrm{nT}$ ) during 1996-2005, J. Geophys. Res. [Space Phys.], 112, A10102, 2007.

Zhukov, A.N., and F. Auchère, On the nature of EIT waves, EUV dimmings and their link to CMEs, $A \& A, \mathbf{4 2 7}, 705-716,2004$.

Cite this article as: Cid C, Palacios J, Saiz E, Guerrero A \& Cerrato Y: On extreme geomagnetic storms. J. Space Weather Space Clim., 2014, 4, A28. 